\title{
Effect of humification during hydrothermal treatment of Aquatic Wastes with inorganic acid
}

\author{
Huijun Zhang ${ }^{1,2, *}$, Nana $\mathrm{Xie}^{1}$, Zihong $\mathrm{Fan}^{1}$, Tengtun $\mathrm{Xu}^{1}$, and Chunhong Tang ${ }^{1,2}$ \\ ${ }^{1}$ College of Environmental Resources, Chongqing Technology and Business University, Chongqing \\ 400067, China \\ ${ }^{2}$ Chongqing Engineering Research Center for Processing, Storage and Transportation of \\ Characterized Agro-Products, Chongqing 400067, China
}

\begin{abstract}
Taking aquatic processing waste as the main experimental object, to explore the effect of different treatment conditions on the humification process in the acid-hydrothermal reaction process, through the analysis of the hydrothermal process of $\mathrm{pH}$ value, organic matter and humus content, humification rate, and infrared spectrum analysis verification to speculate the formation mechanism of humus. The results show that hydrothermal treatment can promote the formation of humus to a certain extent. Under the working conditions of $195^{\circ} \mathrm{C}$ and $80 \mathrm{~min}$, the humus content of aquatic wastes reaches $78.1 \%$ and the humification rate reaches 0.35 . In addition, within a certain treatment intensity, the hydrothermal humification intensity will increase with the increase of reaction time and temperature. Under the same working conditions mentioned above, adding a certain amount of inorganic acid for hydrothermal treatment, the humus content is slightly higher than that of the group without acid. The humification rate reaches 0.45 , which is 0.3 times higher than that of the group without acid. It shows that hydrothermal treatment can promote the formation of humus to a certain extent. Compared with prolonging the reaction time, the increase of temperature has a more obvious effect on the increase of humification rate.

Keywords: Aquatic waste; Hydrothermal treatment; Humification; Humus.
\end{abstract}

\section{Introduction}

Hydrothermal treatment can save the process of dehydration or drying, which is suitable for the treatment of biomass with high water content ${ }^{[1]}$. Compared with extracting a single product, the hydrothermal products of aquatic wastes after hydrothermal treatment can be fully used in agriculture and forestry, which has a better prospect. Some studies have shown that after inorganic acid-hydrothermal treatment, due to the promotion of hydrogen ions in the whole process, the humus content and humification rate have been significantly improved . Therefore, this paper focuses on aquatic wastes, and investigates the effects of reaction temperature, reaction time and inorganic weak acid on the formation and transformation of humus in hydrothermal process. Investigate the acid-base behavior in hydrothermal processing of aquatic wastes.FTIR scan to assess humification process of acid-hydrothermal

*Corresponding author: zhanghj@ctbu.edu.cn 
(acid-HT) after treatment. Study the relationships between water-soluble carbohydrate and phenol fractions (with more attention to the polyaromatic hydrocarbons concentration) and the humification indices of such aquatic wastes during the treatment process. The aim is to study the humification process and mechanism of aquatic wastes in hydrothermal process, and to provide guidance for subsequent research and application.

\section{Materials and methods}

The experimental raw materials are aquatic wastes (such as fish head, viscera, skin, ). Rinse the raw materials repeatedly with deionized water. After cleaning, put the raw material samples into a cooking machine and smash them, and then put them into sealed bags for later use. The remaining samples are frozen $\left(-4^{\circ} \mathrm{C}\right)$ for storage. The principal components of raw material samples are determined in Table 1. Table 1 - Compare these findings (especially humic acids) with the change of structural characterization of the hydrothermal-humification of aquatic wastes.

Table 1. Characteristics of the Dry Aquatic Waste.

\begin{tabular}{lllll}
\hline $\begin{array}{l}\text { Moisture } \\
\text { content } \%\end{array}$ & $\begin{array}{l}\text { Organic } \\
\text { content } \%\end{array}$ & $\begin{array}{l}\text { Humification } \\
\text { content } \%\end{array}$ & Humification riton & $\mathrm{pH}$ \\
\hline $80.5 \pm 0.13$ & $85 \pm 1.83$ & $8.25 \pm 0.27$ & $0.096-0.135$ & 6.75 \\
\hline
\end{tabular}

Note: The content of organic matter is the ratio of the mass of organic matter to the total mass of dry basis; Humus content: the ratio of humus mass to total mass on dry basis; Humification rate is the ratio of humic acid content to fulvic acid content.

The hydrothermal treatment device used in the experiment is a $1 \mathrm{~L}$ autoclave $(\mathrm{FCF}-1$, Gongyi City Yingyu Boyan Instrument and Equipment Factory), and the centrifugal equipment is (TG165, Shanghai Luxiang Instrument). Freeze-drying (LGJ-18A, Beijing Sihuan Scientific Instrument); Total organic carbon analyzer (TOC-L-CPH, Shimadzu Instrument); Fourier transform infrared spectrometer (IRPrestige-21, Shimadzu Instrument).

The conditions of hydrothermal treatment are reaction temperature of $165^{\circ} \mathrm{C}, 180^{\circ} \mathrm{C}$ and $195^{\circ} \mathrm{C}$ respectively. The treatment time are $20,40,60$ and 80 minutes, and acid-hydrothermal treatment (Acid-HT) with $0.01 \%$ sulfuric acid. The heated sample is centrifugally dehydrated, dried in a drying oven at $40^{\circ} \mathrm{C}$ for 1 hour and dried at $-47^{\circ} \mathrm{C}$ for 24 hours in low temperature vacuum (LGJ-18A type), and then analyzed. The content of organic matter was determined by potassium dichromate method in NY525-2012 standard. Humus is determined by sodium pyrophosphate extraction step by step, and its total carbon content is determined by potassium dichromate volumetric method, and the content of humic acid and fulvic acid is measured. In this paper, the humic rate is defined as the ratio of humic acid content to fulvic acid content.

\section{Results and discussion}

\subsection{Changes of $\mathrm{pH}$ by hydrothermal treatment}

Figure 1 shows the change of $\mathrm{pH}$ of products under different hydrothermal conditions. In the process of hydrothermal reaction, the macromolecular organic matter in the raw materials of aquatic waste samples breaks the bond, and some acidic groups such as carbon group, carboxyl group, aldehyde group and phenolic hydroxyl group are generated. At the same time, during the reaction, a part of the generated organic acids enter the solution, which makes the solution after the reaction acidic. It can be seen from Figure 1 that when the reaction time reaches 20 minutes, the $\mathrm{pH}$ of the solution is already acidic. However, when the reaction time of acid-hydrothermal treatment (acid-HT) reached 60 minutes, the $\mathrm{pH}$ of the solution slightly 
increased after the reaction, which indicated that some organic acids in the product were decomposed at this time. This made the $\mathrm{pH}$ of the solution increase. On the whole, however, with the increase of temperature and the extension of time, the $\mathrm{pH}$ of the solution after the reaction decreased to varying degrees, which indicated that with the intensification of hydrothermal degree, the primary hydrolysis reaction of organic matter became more intense. Easily degradable organic matter was decomposed under the promotion of hydrogen ions of water self-ionization, and organic acids were generated. At the same time, the generated organic acids promoted the hydrolysis reaction, and the $\mathrm{pH}$ of the solution after the reaction showed a downward trend. This downward trend is especially obvious when the reaction temperature is $195^{\circ} \mathrm{C}$.

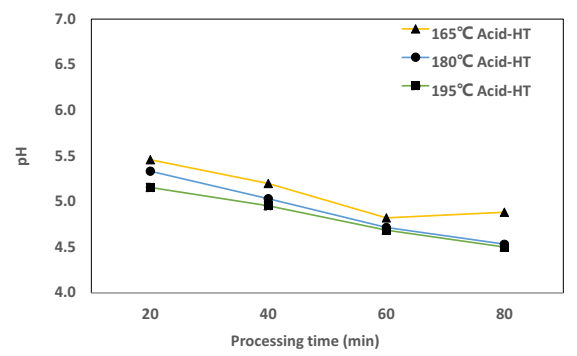

Fig .1. pH of products under different hydrothermal conditions.

\subsection{Changes of organic matter by hydrothermal treatment}

Figure 2 shows the change of organic matter under different hydrothermal conditions. From the figure, it can be seen that in the initial hydrolysis stage of hydrothermal reaction, there is obvious loss of organic matter. The reason is that in this stage, acromolecules are decomposed into small molecules and dissolved in the liquid phase. With the extension of treatment time, the hydrothermal reaction went to the second stage, and some small molecules condensed with aromatic compounds to generate various new organic matters ${ }^{[2]}$. Therefore, the content of organic matter began to show an upward trend again. It can also be seen from Figure 2 that in acid-hydrothermal treatment (acid-HT) at $165^{\circ} \mathrm{C}$, when the hydrothermal treatment time is 40-60 minutes, the organic matter content always shows a slow downward trend, while at $180^{\circ} \mathrm{C}$ and $195^{\circ} \mathrm{C}$, the organic matter content begins to show an upward trend after 40 minutes of hydrothermal reaction. This shows that the increase of temperature can accelerate the decomposition reaction at the initial stage of hydrothermal treatment. It can also be found from the figure that there is a certain loss of organic matter after hydrothermal treatment, but the loss is relatively small, and the content of organic matter reaches $79.1 \%$ at $165^{\circ} \mathrm{C}$ and $20 \mathrm{~min}$, which is $45 \%$ higher than the national standard of organic fertilizer agriculture.

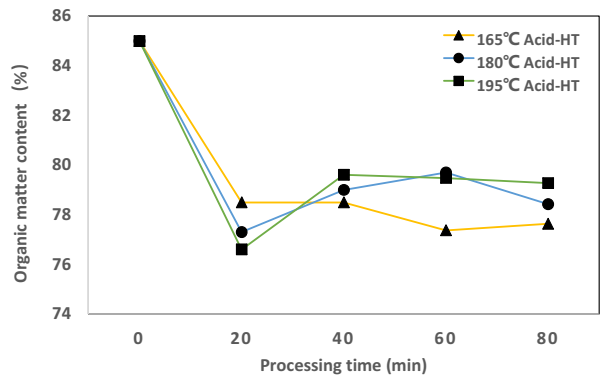

Fig. 2. Organic matter content of products under different hydrothermal conditions. 


\subsection{Effect of hydrothermal treatment on humification process}

Figure 3 shows the influence of hydrothermal treatment on humification process. In this paper, the humification rate is defined as the ratio of humic acid content to fulvic acid content. Although there is no corresponding evaluation standard for humification rate in hydrothermal treatment, hydrothermal products are similar to compost products, this paper evaluates the maturity of hydrothermal process with reference to the evaluation standard of compost. It is generally believed that when the humification rate is greater than $>0.8$, the compost can be considered as mature ${ }^{[3]}$. The humic rate of raw material samples is 0.096 , and the degree of maturity is extremely low. Acid-hydrothermal treatment (acid-HT) has a humification rate of 0.35 at $180^{\circ} \mathrm{C}$ for $60 \mathrm{~min}$. With the increase of temperature to $195^{\circ} \mathrm{C}$, the humification rate of hydrothermal products increases more obviously. When the reaction time reaches $60 \mathrm{~min}$ and $80 \mathrm{~min}$, the humification rate is 0.44 and 0.45 respectively. Figure 3 shows that under six hydrothermal conditions, the humification rate does not meet the requirement of the evaluation standard that the humification degree is greater than 0.8 , but it is increased by about 2-4 times compared with the humification rate of raw materials, which shows that hydrothermal treatment can promote the formation of humus to some extent.

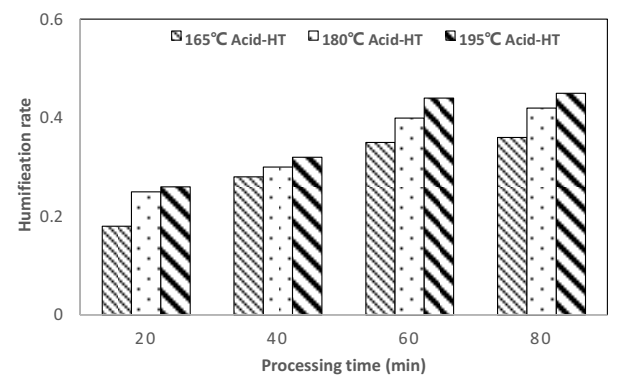

Fig. 3. Humifieation rate of products under different hydrothermal conditions.

\subsection{Infrared spectrum analysis}

By searching the spectrogram ${ }^{[4]}$, it is recognized that the infrared spectrogram drawn by the acid-hydrothermal (acid-HT) at $165^{\circ} \mathrm{C}$ for 80 minutes belongs to the following absorption peaks: one absorption peak of aromatic substances (benzene skeleton stretching vibration at $1516.40 \mathrm{~cm}-1)$ and two absorption peaks of sugar substances (OH stretching vibration of sugar $\mathrm{C}-\mathrm{OH}$ at $448.79 \mathrm{~cm}^{-1}$; stretching vibration of sugar $\mathrm{C}-\mathrm{OH}$ at $1016.56 \mathrm{~cm}^{-1}$ ).

Through the comparison of spectrogram retrieval, it is recognized that the absorption peaks of each region in the infrared spectrogram drawn by the acid-hydrothermal (acid-HT) at $195^{\circ} \mathrm{C}$ for 80 minutes belong to the following: four absorption peaks of aromatic substances (Aromatic aldehyde stretching vibration at $1,726.65 \mathrm{~cm}-1$; aromatic $\mathrm{C}=\mathrm{C}$ telescopic vibration at $1,616.49 \mathrm{~cm}-1$; benzene-like skeleton stretching vibration at 1,508.41 $\mathrm{cm}-1$; symmetric stretching vibration of aromatic acid ester $\mathrm{C}-\mathrm{O}-\mathrm{C}$ at $1036.63 \mathrm{~cm}-1), 1$ carbohydrate absorption peak (OH stretching vibration of sugar $\mathrm{C}-\mathrm{OH}$ at $3446.79 \mathrm{~cm}-1), 1$ absorption peak of phenolic alcohols (-OH Telescopic Vibration of Phenol Hydroxyl at $3417.79 \mathrm{~cm}-1)$.

From the above analysis, it can be seen that the products under hydrothermal conditions at $195^{\circ} \mathrm{C}$ for 80 minutes contain more aromatic functional groups than those under hydrothermal conditions at $165^{\circ} \mathrm{C}$ for 80 minutes. This shows that with the deepening of the reaction degree, the formation of macromolecular humic acid becomes more intense, so the aromatic compounds in the product increase. This also shows that hydrothermal reaction can 
promote the formation of humus to a certain extent, and aquatic wastes can gradually mature with the increase of temperature in hydrothermal reaction. which is consistent with the above inference results (3.3).

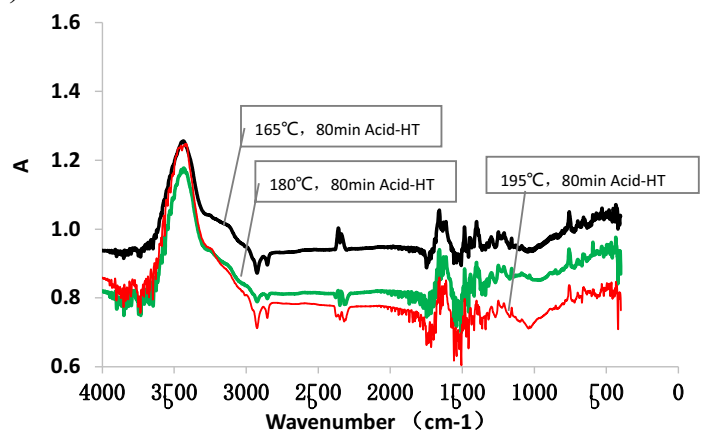

Fig. 4. The infrared spectra different hydrothermal conditions.

\section{Conclusion}

In the actual working condition, the humification rate of acid-water heat treated samples at $195^{\circ} \mathrm{C}$ for $80 \mathrm{~min}$ is 0.45 , which only reaches $50 \%$ of the requirement of the evaluation standard that the degree of humification is $>0.8$, but compared with the humification rate of raw materials, it is increased by about 2-4 times, indicating that hydrothermal treatment can promote the formation of humus to a certain extent. The experimental results show that, compared with prolonging the reaction time, the increase of temperature has a more obvious effect on the increase of humification rate.

Funding: National Natural Science Foundation of China [Project No.51808079], the natural science foundation of Chongqing [Project No. cstc2017jcyjAX0470 and cstc2020jscx-lyggX0001] and Chongqing Technology and Business University Scientific Research Foundation [Project No. 670101379 and CQCM-2016-07].

\section{References}

1. Dewatering, Dehydration and drying of sludge. Anaerobic Sewage Treatment: Optimization of Process and Physical Design of Anaerobic and Complementary Processes, 2019, pp.257-291.

2. Evaluation of physicochemical characteristics and health risk of polycyclic aromatic hydrocarbons in borehole waters around automobile workshops in Southeastern Nigeria. Groundwater for Sustainable Development 14, 100615. 2021.

3. Mahmoud I B, Medhioub M, Rigane H, et al. Evolution of humicfractions in calcimagnesic soils and brown isohumic soils amended with compost and manure[J]. Compost Science\&Utilization, 2007,15(4):253

4. S Wenger Shi-Pu, Fourier transform infrared spectrum analysis, Chemical Industry Press (CIP) 2004. 